\title{
Detect or not to detect very early stage hepatocellular carcinoma? The western perspective
}

\author{
Ju Dong Yang ${ }^{1,2,3,4}$ \\ 'Division of Digestive and Liver Diseases, Department of Medicine, ${ }^{2}$ Comprehensive Transplant Center, and ${ }^{3}$ Samuel Oschin \\ Comprehensive Cancer Institute, Cedars Sinai Medical Center, Los Angeles, CA; ${ }^{4}$ Division of Gastroenterology and Hepatology, \\ Mayo Clinic, Rochester, MN, USA
}

\begin{abstract}
Very early stage hepatocellular carcinoma $(\mathrm{HCC})$ is defined as a single tumor with the largest diameter of the lesion measuring $2 \mathrm{~cm}$ or less according to Barcelona Liver Cancer staging system. Detection of very early stage HCC is clinically important as it confers an excellent prognosis with the 5-year survival rates over 60 to $80 \%$ after patients receive curative treatments. While diagnosing HCC at a very early stage is crucial, it is technically challenging and may come with the physical or psychosocial harms related to diagnostic tests. It is further complicated by the fact that patients with very early stage HCC are not prioritized for liver transplant (LT) in the United States organ allocation system. When LT-eligible patients present with an indeterminate lesion measuring between 1 and $2 \mathrm{~cm}$ on the multiphasic computed tomography or magnetic resonance imaging, clinicians often observe patients carefully until the lesion grows up to $2 \mathrm{~cm}$ so that patients can be eligible to receive a Model for End-Stage Liver Disease (MELD) exception score for HCC in the United States. The European guideline recommends a routine biopsy of such lesion. In conclusion, attempting to detect very early stage HCC is difficult to achieve and controversial. Clinicians should take into account of the risk and the benefit of diagnostic tests, LT candidacy of patients and the local organ allocation system. (Clin Mol Hepatol 2019;25:335-343)
\end{abstract}

Keywords: Liver cancer; Hepatocellular cancer; Diagnosis; Hepatocellular carcinoma (HCC)

\section{INTRODUCTION}

Early detection of hepatocellular carcinoma (HCC) is important as a tumor stage at the time of diagnosis is closely related to the clinical outcomes. ${ }^{1,2}$ While patients with very early stage HCC have excellent clinical outcome, diagnosing cancer at a very early stage is technically challenging. Noninvasive diagnosis of HCC is established by characteristic radiologic features in the liver specific mul- tiphasic computed tomography (CT) or magnetic resonance imaging (MRI) in cirrhotic patients. ${ }^{3-5}$ Attempting to establish HCC diagnosis at a very early stage may come with risks. For instance, biopsy could be considered in further interrogation of indeterminate lesions on CT or MRI. It can cause complications, including but not limited to bleeding and needle track tumor seedings. ${ }^{6,7}$ Although biopsy is highly specific for HCC diagnosis, but it is not sensitive for the diagnosis of very early stage HCC due to difficul-

\footnotetext{
Abbreviations:

AASLD, the American Association for the Study of Liver Diseases; AFP, alphafetoprotein; ALT, alanine aminotransferase; AUC, area under the curve; BCLC, Barcelona Liver Cancer; CEUS, contrast-enhanced ultrasound; CT, computed tomography; ctDNA, circulating tumor DNA; CTP, Child-Turcotte-Pugh; DCP, desgamma-carboxy prothrombin; EASL, the European Association for the Study of the Liver; HBV, hepatitis B virus; HCC, hepatocellular carcinoma; HCV, hepatitis C virus; iCCA, intrahepatic cholangiocarcinoma; LI-RADS, Liver Imaging Reporting And Data System; LT, liver transplant; MELD, Model for End-Stage Liver Disease; MRI, magnetic resonance imaging; US, ultrasonography
}

\section{Corresponding author : Ju Dong Yang}

Division of Digestive and Liver Diseases, Department of Medicine, Cedars Sinai Medical Center, 8900 Beverly Blvd, Los Angeles, CA 90048, USA

Tel: +1-310-423-1971, Fax: +1-310-423-2356

E-mail:judong.yang@cshs.org

https://orcid.org/0000-0001-7834-9825 
ties with accurate targeting of the small lesion. ${ }^{8}$ Furthermore, patients with very early stage HCC are not eligible to receive a Model for End-Stage Liver Disease (MELD) exception score in the current organ allocation system in the United States, hampering the enthusiasm for detection of HCC at a very early stage especially in the liver transplant (LT) candidates. ${ }^{9}$ This article discussed the definition of very early stage $\mathrm{HCC}$, rationales and challenges in attempting to detect tumors at a very early stage, recall policy for indeterminate liver nodule and implication of the United States organ allocation system in the clinical practice.

\section{DEFINITION OF VERY EARLY STAGE HCC}

While a number of staging systems have been proposed for $\mathrm{HCC}$, only few of them have specific definition of very early stage of HCC. ${ }^{10,11}$ Very early stage of HCC has clearly distinct clinical characteristics in diagnosis and prognosis and detection of cancers at a very early stage is a goal of liver cancer surveillance program in high risk individuals. The Barcelona Liver Cancer (BCLC) classification is a standard staging system endorsed by the American Association for the Study of Liver Diseases (AASLD) and the European Association for the Study of the Liver (EASL). ${ }^{3,412}$ ACcording to the BCLC staging system, very early stage HCC is defined as a single tumor with the largest diameter of $2 \mathrm{~cm}$ or less, well-preserved liver function without vascular invasion or metastasis. ${ }^{10}$ Patients with very early stage HCC are recommended to undergo ablation or resection for potentially curative treatments and their outcome is excellent with the 5 -year overall survival over 60-80\%. ${ }^{13,14}$ ITA.LI.CA tumor staging system also had the same definition for very early stage HCC, namely, a single nodule and 2 $\mathrm{cm}$ or less in size from the extent of tumor standpoint. ${ }^{11,15}$

\section{RATIONALE FOR DETECTING VERY EARLY STAGE HCC}

Detection of very early stage HCC is crucial as patients presenting at this stage have excellent prognosis. ${ }^{13,14} \mathrm{~A}$ multicenter surgical series of 132 patients in the United States and Italy showed the median survival of 75 months with the 5-year survival rate of $70 \%$ after surgical resection of $\mathrm{HCC}^{13}$ Japanese nationwide survey of patients who underwent curative treatments for very early stage HCC with preserved liver function (Child-Turcotte-Pugh [CTP] class A) had the 5 -year survival of $84 \%$ after surgical resec- tion $(n=785)$ and $77 \%$ after percutaneous ethanol ablation $(n=543))^{14}$ The study demonstrated that the size of the lesions is closely associated with the prognosis of patients after curative treatment even when the size of the tumor is still small. ${ }^{14}$ The 5 -year overall survival rate decreased by $10 \%$ after curative resection or ablation once tumor size was between 2-3 cm compared to survival probability of patients with tumor size $2 \mathrm{~cm}$ or less, highlighting the clinically meaningful superiority in overall survival in patients with a very early stage $\mathrm{HCC}$ over an early stage $\mathrm{HCC}{ }^{14}$

Attempting to diagnose a very early stage HCC may also help clinicians to find different types of liver cancer, most commonly intrahepatic cholangiocarcinoma (iCCA), which can affect transplant candidacy of the patients and their long-term outcome. For instance, several studies showed that a small subgroup of patients with iCCA could potentially benefit from LT. ${ }^{16-18}$ A recent retrospective multicenter study of 81 patients from 17 LT centers showed that patients with a very early stage iCCA (single nodule and $2 \mathrm{~cm}$ or smaller in the tumor size) at explant had the 5 -year post-transplant tumor recurrence rates of $18 \%$ and the 5 -year overall survival rates of $65 \% .{ }^{16}$ On the other hand, patients with beyond very early stage disease, of whom $80 \%$ were still within Milan criteria, had the 5-year post-transplant recurrence rates of $61 \%$ and the 5 -year overall survival rates of $45 \%$. Author also reported an outcome of group of patients with single tumor, size between 2.1 and $3 \mathrm{~cm}$ without poor differentiation on histology. The 5 -year overall survival rates were $61 \%$, which is within acceptable range for LT. Given promising outcome of selected subgroup of patients with small solitary iCCA after LT, several transplant centers adopted a LT protocol for iCCA. The iCCA protocol at the Mayo clinic was summarized in Table 1. Currently, most transplant center in the United States would not consider LT in patients with multifocal iCCA even if patients present within Milan criteria due to poor post-LT outcomes.

\section{DIAGNOSTIC CHALLENGES}

Diagnosing a very early stage HCC is challenging to achieve in the western population. A previous study showed that up to 15 to $20 \%$ of HCC seen in the explant were incidental finding, meaning that these tumors were not recognized in routine pre-transplant liver images. ${ }^{19} \mathrm{~A}$ recent multicenter study showed that almost half of the patients with a very early stage iCCA (46.7\%) had been transplanted without pre-transplant radiologic recognition of a suspicious hepatic nodule. One study showed that underlying eti- 
ology of alcohol or hepatitis C virus (HCV), old age, and increased alpha-fetoprotein (AFP) were associated with presence of incidental HCC. ${ }^{20}$

Liver ultrasound is a standard surveillance imaging test for HCC. ${ }^{3,4}$ However, quality of liver ultrasound as a surveillance test is often limited due to high prevalence of obesity and metabolic liver disease in the western countries. ${ }^{21,22} \mathrm{~A}$ recent retrospective multicenter study evaluated the quality of ultrasonography (US) image as a surveillance test. Radiologists reviewed all ultrasound exams in 941 cirrhosis patients and $20 \%$ of ultrasound images were inadequate for excluding HCC. Male gender, body mass index, CTP B or C cirrhosis, alcohol-related and non-alcoholic steatohepatitis-related cirrhosis were independently associated with inadequate US. ${ }^{22}$ A recent meta-analysis showed that ultrasound alone has $47 \%$ sensitivity for detection of early stage HCC. Although there was an increase in sensitivity in the recent era $(50 \%$

Table 1. Liver transplant criteria for intrahepatic cholangiocarcinoma at Mayo Clinic

\begin{tabular}{l}
\hline Biopsy proven unresectable intrahepatic cholangiocarcinoma \\
Single lesion $\leq 3 \mathrm{~cm}$ in diameter \\
No macrovascular invasion \\
No microvascular invasion \\
No poorly differentiated histology \\
No evidence of extrahepatic disease \\
No attempted prior resection with violation of tumor plane \\
Reasonable candidate for liver transplant
\end{tabular}

Patients should meet all listed criteria. for study conducted after 2000), it did not reach a statistical significance. ${ }^{23}$ Due to limited sensitivity of liver US for detection of HCC, cross-sectional images have been entertained as a surveillance test. Four phasic $\mathrm{CT}$ or dynamic MRI are essential non-invasive diagnostic modalities for $\mathrm{HCC}^{24,25}$ Diagnosis is achieved when the lesion is at least $1 \mathrm{~cm}$ or larger in size with characteristic enhancement patterns, namely arterial phase hyperenhancement and washout in the portal venous or delayed phase on CT or MRI (Fig. 1). ${ }^{3,26}$ More recently, AASLD adopted the Liver Imaging Reporting And Data System (LI-RADS) for non-invasive diagnosis of HCC, which was originally developed to standardize radiological diagnosis and reporting, define minimum imaging technical requirements, and create a mechanism for formal data collection in patients at risk for $\mathrm{HCC}^{27,28}$ According to the most recent LIRADS, size of the lesion affects the diagnostic criteria for HCC. For lesion $\geq 2 \mathrm{~cm}$, non-rim arterial phase hyperenhancement and one more feature (enhancing capsule, non-peripheral washout, or threshold growth) are diagnostic for HCC. For lesion between 1 and $2 \mathrm{~cm}$, non-rim arterial phase hyperenhancement and one more feature (non-peripheral washout or threshold growth) are diagnostic for HCC (Fig. 1). ${ }^{28}$ The non-invasive criteria should be applied only in patients with underlying cirrhosis of the liver given high pretest probability. One positive test is enough to confirm the diagnosis. $^{29}$

Several studies compared the diagnostic performance of multiphasic CT, MRI and liver US for detection of HCC. ${ }^{29-33}$ One study reviewed CT or MRI features in the 101 nodules (34\% were malignant lesions) of 84 cirrhosis patients. The sensitivity of $\mathrm{CT}$ and

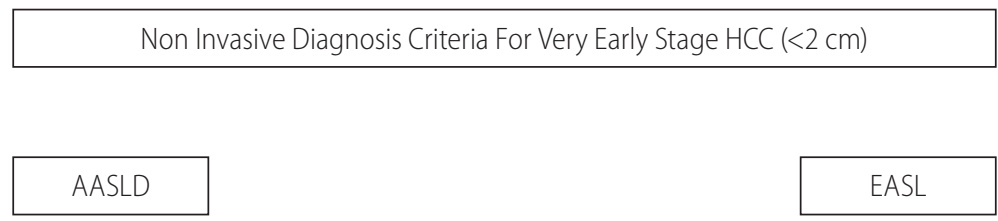

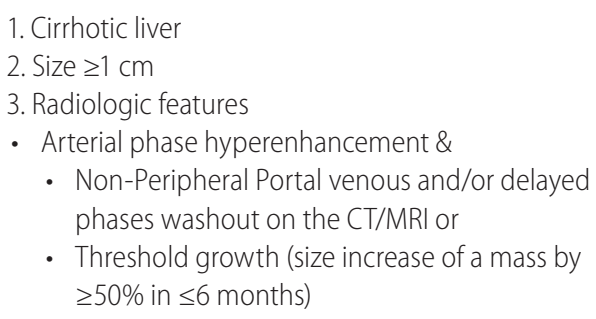

1. Cirrhotic liver

2. Size $\geq 1 \mathrm{~cm}$

3. Radiologic features

- Arterial phase hyperenhancement \&

- Portal venous and/or delayed phases washout on the CT/MRI or

- Late washout (>60 seconds) of mild degree on the contrast enhanced US

Figure 1. Noninvasive diagnosis criteria for very early stage hepatocellular carcinoma (HCC). Modified from European Association for the Study of the Liver $^{3}$ and Marrero et al. ${ }^{4}$ AASLD, the American Association for the Study of Liver Diseases; EASL, the European Association for the Study of the Liver; $\mathrm{CT}$, computed tomography; MRI, magnetic resonance images; US, ultrasonography. 
MRI was only $53 \%$ and $62 \%$, respectively for the diagnosis of HCC. A recent study investigated the performance of MRI with liver-specific contrast (gadoxetic acid) for detection of HCC in the surveillance setting. ${ }^{32}$ This study analyzed 407 high-risk cirrhosis patients (estimated annual risk of HCC development $>5 \%$ ) who underwent 1,100 surveillance tests with paired US and MRI. The HCC detection rate was much higher with MRI (86.0\%) than US (27.9\%). MRI showed a significantly lower rate of false-positive findings than US. Of the 43 patients with HCC, 32 (74.4\%) had very early stage HCC, suggesting that MRI with liver-specific contrast may be an excellent surveillance test although it still missed to detect very early stage HCC in a quarter of the patients. Overall, MRI appears to have a higher sensitivity in detecting HCC over $\mathrm{CT}$ scan. ${ }^{34} \mathrm{~A}$ recent meta-analysis showed a pooled sensitivity of $82 \%$ and $66 \%$ for MRI and $\mathrm{CT}$ for detection of HCC, respectively $(P<0.01))^{34} \mathrm{~A}$ subgroup analysis showed a numerically higher sensitivity of $76 \%$ for MRI than $68 \%$ for $\mathrm{CT}$ for the detection of hepatic lesion less than $2 \mathrm{~cm}$, but it did not reach a statistical significance. $^{34}$ Routine use of MRI or CT scan could be considered to increase the probability of capturing small lesions less than $2 \mathrm{~cm}$, but it can pose physical or psychosocial harm and may not be cost-effective. ${ }^{35}$ However, a recent Markov modeling study suggested that using cross-sectional images could be cost-effective in high-risk patients. The study showed that MRI is a cost-effective surveillance test in high-risk HCC patients with an annual incidence rate of $1.81 \%$ or higher when the incremental cost-effectiveness ratio becomes $\$ 50,000$ or less per one quality-adjusted life-years. ${ }^{36}$

With regard to blood-based diagnostic tests, AFP alone appears to have suboptimal performance for detection of early stage HCC. ${ }^{27,37}$ But it appears to be helpful in detecting early stage HCC as combination of the other diagnostic test. ${ }^{38} \mathrm{~A}$ recent study showed that AFP is an independent risk factor of HCC development in cirrhotic patients with indeterminate nodules $(<2 \mathrm{~cm})$ detected on the CT scan. ${ }^{39}$ It should be noted that AFP levels can be falsely elevated in patients with underlying hepatic inflammation, particularly with hepatitis B virus (HBV) or C virus infection, but appear to be an excellent biomarker in patients with normal alanine aminotransferase (ALT). ${ }^{40,41}$ Several studies showed that longitudinal measurement of tumor markers might be helpful for the detection of HCC too. ${ }^{42,43}$ AFP-L3\% and des-gamma-carboxy prothrombin (DCP) appear to have complementary roles for detection of $\mathrm{HCC}^{27,44} \mathrm{~A}$ recent phase 3 diagnostic biomarker study showed that the combination of AFP (5 ng/mL) and AFP-L3 (4\%) provide a sensitivity and specificity of $79 \%$ and $87 \%$ for detection of HCC, respectively. ${ }^{42}$ Over $90 \%$ had HCC within Milan criteria and $74 \%$ had very early stage HCC in that study. ${ }^{42}$ HBV $(79 \%)$ was the leading etiology of HCC and most patients had normal ALT (87\%), due to successful control of viral replication with potent antiviral treatment. Overall performance of AFP L3\% was superior to liver ultrasound whose sensitivity was only $49 \%$. A recent United States study showed that the GALAD score, a composite of demographic risk factors (age and male gender) and three serum-based biomarkers (AFP, AFP-L 3\% and DCP) might be a promising risk score for detection of very early stage HCC with the sensitivity of $100 \%$ at the specificity of $79 \%$ with the area under the curve (AUC) of $0.91 .{ }^{21}$ A multicenter study from UK, Germany and Japan showed similar results with the AUC ranging between 0.89 and 0.92 for detection of very early stage $\mathrm{HCC}^{45}$ More recently, improvement of next-generation sequencing technology and better understanding of genetic or epigenetic alteration of HCC have allowed comprehensive analysis of mutational and methylation landscape of circulating nucleic acid-based biomarkers. Circulating cell free DNA (cfDNA) is a fragmented DNA subtype, found in the blood circulation. Circulating tumor DNA is the fraction of total cfDNA, which originates from the primary tumor or metastases in patients with cancer. One study reported 6-marker cfDNA methylation panel and it yielded the $95 \%$ sensitivity and the $92 \%$ specificity with the AUC of 0.96 for $\mathrm{HCC}$ detection. ${ }^{46}$ Of note, the cfDNA methylation panel detected $75 \%$ of very early stage $\mathrm{HCC}$ and $93 \%$ of patients meeting Milan criteria for $\mathrm{HCC}^{46}$ So far, none of circulating nucleic acid-based biomarkers have yet been validated in longitudinal cohorts for pre-clinical detection of HCC. Overall, data in the literature highlight the limited sensitivity of current radiologic and blood-based tests for very early stage HCC detection and fine characterization of these small lesions.

\section{LIVER TRANSPLANT FOR HCC; ORGAN ALLO- CATION HISTORY IN THE UNITED STATES}

LT is a potentially curative treatment option for HCC. While it is a highly resource intensive treatment, it is the most definitive treatment for HCC as it removes not only the tumor, but also the diseased liver, which may contain foci of microscopic metastasis or serve as nidus for the development of de novo $\mathrm{HCC}{ }^{47}$ Hence, recurrence rate of HCC after LT is less than $15 \%$, which is substantially lower than $70 \%$ recurrence rate after other curative treatments such as resection or ablation. ${ }^{13,14,48}$ For this reason, LT has been increasingly utilized for the treatment of HCC and a re- 
cent study showed that HCC is the leading indication for LT listing and surgery in the United States. ${ }^{49}$

The MELD score was developed to predict 3-month mortality in patients with end-stage liver disease and was adopted by United Network for Organ Sharing for organ allocation. ${ }^{50,51}$ Patients listed for LT with HCC typically have lower MELD scores than patients listed without HCC, thus MELD score underestimated the risk of mortality on the waitlisted HCC patients. Thus, MELD exception point was granted in HCC patients since 2002 if the tumors were within Milan criteria (one lesion less than $5 \mathrm{~cm}$ or up to 3 lesions with the largest lesion less than $3 \mathrm{~cm}$ ). As patients with larger tumor burden had an increased risk of tumor progression and subsequent dropout of the listing, patients with $\mathrm{T} 2$ disease (1 lesion between 2 and $5 \mathrm{~cm}$ or $2-3$ lesions with none larger than $3 \mathrm{~cm}$ ) received a MELD exception score of 29. Those with T1 (solitary lesion $<2 \mathrm{~cm}$ ) disease received 24 instead of 29 as their risk of tumor progression and likelihood of subsequent dropout of the listing and death was lower than those with T2 disease. However, concerns were raised that patients with HCC were advantageous due to excessive exception point. To address this limitation, several revisions were made for MELD exception policy for HCC to achieve the balance of benefit between HCC and non-HCC patients. ${ }^{9}$ HCC MELD exception point has been decreasing over time since and no exception point was granted for T1 lesions since 2004. Most recent change was made in 2015 and patient should now remain on their biologic MELD score for the first 6 months after listing and patients are granted MELD score of 28 after 6 months of waiting on the list with the cap MELD of $34{ }^{52}$

Lack of MELD exception point for T1 lesion has made a huge impact on the approach for detection of very early stage HCC especially in patients who are eligible for $L T$ as patients with very early stage HCC are no longer prioritized for LT in the United States organ allocation system. Patients with very early stage HCC often have to wait until tumor grows beyond the very early stage when they are eligible to receive MELD exception score. Patients with indeterminate small lesions on diagnostic images (multiphasic CT or MRI) often have been closely followed up without percutaneous biopsy of the liver lesion. A recent study from University of California, San Francisco investigated the outcome of the "wait and not ablate" approach in 114 patients with T1 HCC (1 lesion $<2 \mathrm{~cm}$ ). ${ }^{53}$ Patients were monitored with cross-sectional images every 3 months. Vast majority of HCC patients (88\%) had stage progression from T1 to T2 (1 lesion $2-5 \mathrm{~cm}$ or $2-3$ lesions $\leq 3 \mathrm{~cm}$ ) at a median duration of 6.9 months. Only six (5.3\%) patients progressed from T1 directly to beyond T2 criteria at a median dura- tion of 5.1 months. ${ }^{53}$ While tumor biology may change with the progression of disease, the probability of tumor progression appears to be low enough that most transplant centers favor close observation in this setting rather than subjecting patients to percutaneous biopsy in otherwise good candidate for LT. For patients who would not be a good candidate for LT, attempting to establish a very early stage HCC is crucial to improve clinical outcome. Percutaneous biopsy is often performed followed by a local ablation in such patients with indeterminate lesion on CT or MRI measured between 1 and $2 \mathrm{~cm}$.

\section{RECALL POLICY FOR INDETERMINATE LIVER MASS ON SURVEILLANCE ULTRASOUND}

In the setting of HCC surveillance, liver ultrasound may show small indeterminate lesions. The algorithm to be followed abnormal surveillance tests is called a recall policy. A recall policy is essentially the same between the AASLD and the EASL when the lesion is less than $1 \mathrm{~cm}$ in size (Fig. 2). ${ }^{3,4}$ Probability of these lesions measuring $1 \mathrm{~cm}$ or less representing HCC is very slim. ${ }^{8}$ Therefore, diagnostic image is not recommended but follow up liver ultrasound is recommended between 3 and 6 months. The recall policy is somewhat different when the lesion is between 1 and $2 \mathrm{~cm}$ between the AASLD and the EASL. The AASLD and the EASL both recommend that the lesions be further characterized by multiphasic liver-specific CT or MRI. The AASLD adopted LIRADS for noninvasive diagnosis of HCC. ${ }^{54}$ LI-RADS system classifies lesions into 7 different categories (Fig. 2). ${ }^{28,55}$ LI-RADS 1 lesions are definitively benign and return to regular surveillance imaging every 6 months is recommended. LI-RADS 2 lesions are probably benign and return to regular surveillance imaging every 6 months is recommended although further diagnostic imaging could be considered in 6 months or less depending on other risk factors. LI-RADS 3 lesions has intermediate probability for HCC and repeat or alternative diagnostic imaging is recommended in 3-6 months. LI-RADS 4 lesions are probably HCC and repeat or alternative diagnostic imaging is recommended in less than 3 months. Biopsy of the LI-RADS 4 lesion could be considered after multi-disciplinary discussion in selected cases. LI-RADS 5 lesions are diagnostic for HCC. LI-RADS added two additional categories. LI-RADS NC refers to non-categorizable lesions due to images omission or severe degradation and LI-RADS M refers to lesions that are consistent with malignancy, but not definitively HCC. A recent meta-analysis reported the pooled percentages of observa- 


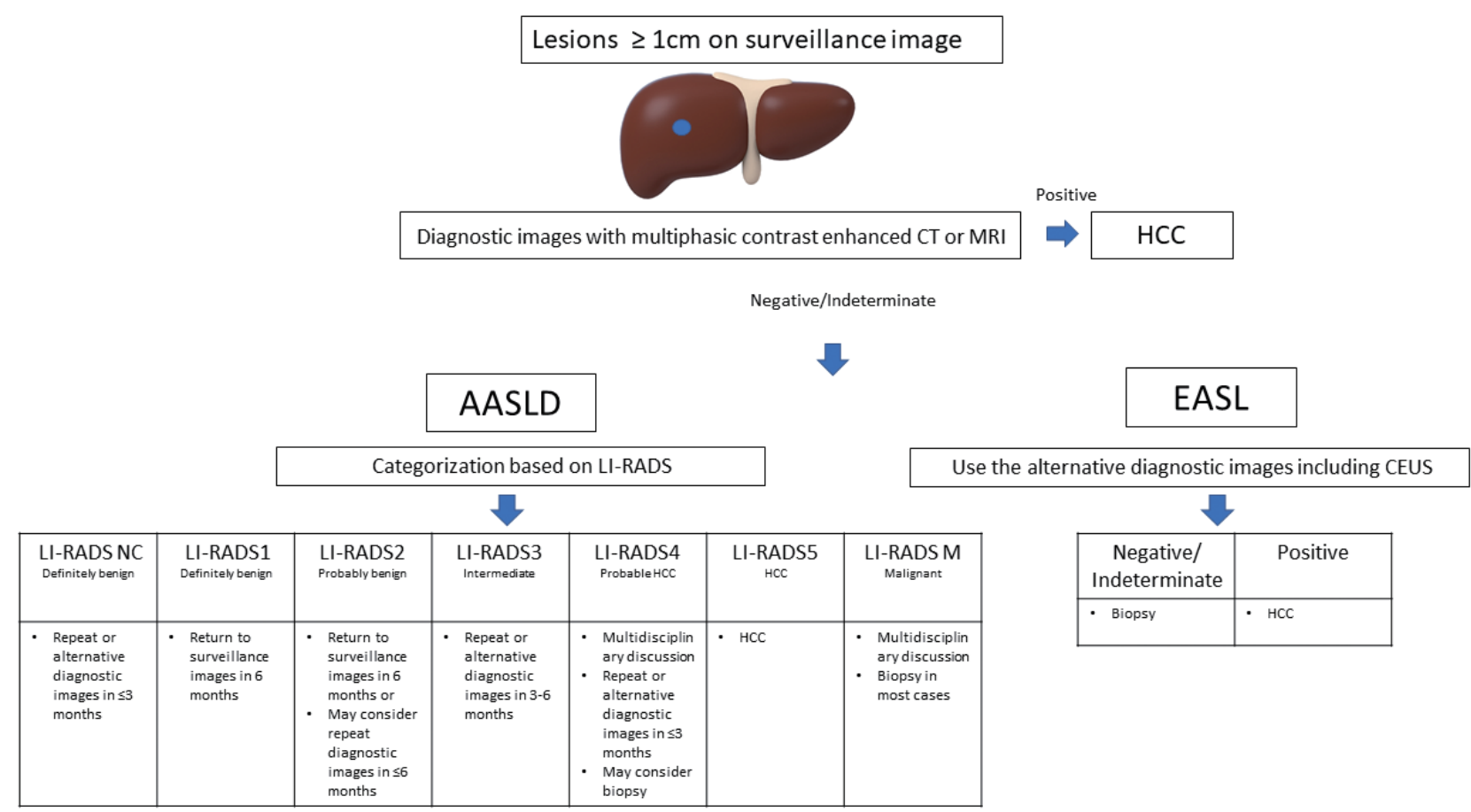

Figure 2. Comparison of recall policy between the American Association for the Study of Liver Diseases (AASLD) and the European Association for the Study of the Liver (EASL) guideline. Adapted from European Association for the Study of the Liver ${ }^{3}$ and Marrero et al. ${ }^{4} \mathrm{CT}$, computed tomography; MRI, magnetic resonance imaging; HCC, hepatocellular carcinoma; LI-RADS, Liver Imaging Reporting And Data System; CEUS, contrast-enhanced ultrasound.

tions confirmed as HCC in 3,556 observations from 2,760 patients, of whom 2,482 had HCCs. ${ }^{56}$ The pooled percentages for the lesions representing HCC were $94 \%$ for LR-5, 74\% for LR-4, $38 \%$ for LR-3, $13 \%$ for LR-2, $0 \%$ for LR-1 and $36 \%$ for the LR-M group. AASLD does not recommend a routine biopsy of indeterminate lesion as it may harm patients with complications including bleeding or tumor seeding ${ }^{6,7}$ However, biopsy would be an excellent diagnostic tool for detection of very early stage HCC especially in selected cases of LI-RADS 4 lesions as it may facilitate early diagnosis of HCC.

The EASL proposes different approaches (Fig. 2). ${ }^{3}$ When a surveillance US shows lesions less than $1 \mathrm{~cm}$, repeat US is recommended in 4 months without diagnostic images. Once the lesion measures $1 \mathrm{~cm}$ or over, diagnostic tests should be performed for further characterization. The EASL recommends a multiphasic CT or MRI as a first-line diagnostic test, but also consider contrastenhanced ultrasound (CEUS) as a diagnostic test. A recent retrospective study showed that characteristic features of CEUS (arterial enhancement followed by late washout, onset $>60$ seconds after contrast injection) have a positive predictive value of HCC at $99 \%$ without misclassification for cholangiocarcinoma. ${ }^{57}$ A recent multicenter prospective study showed that the specificity of CEUS for the diagnosis of HCC in 1-2 cm lesion is $93 \%$, which is higher than $83 \%$ for MRI and $77 \%$ for $\mathrm{CT}^{58}$ However, CEUS has a relatively low sensitivity and cannot examine the entire liver due to very short arterial phase. Its performance may also be affected by body habitus of the patients and location of the lesion. Further, patients will need a staging cross-sectional image once HCC is confirmed. Hence, CEUS is not recommended as a first-line diagnostic images but could be an effective diagnostic test in patients when $\mathrm{CT}$ and MRI are contraindicated or show inconclusive lesions. ${ }^{3}$ If any of three images shows diagnostic features of HCC, then $\mathrm{HCC}$ diagnosis can be secured. However, if any of the three images fail to show characteristic features of HCC, then biopsy is recommended. This contrasts to the recommendation from the AASLD where routine biopsy of such lesions is discouraged. The rationales to establish the diagnosis early on is because delaying it until tumor reaches $2 \mathrm{~cm}$ or larger can increase the probability of treatment failure or recurrence as tumor start developing satellite nodules or microscopic vascular invasion once it transitions from very early stage to early stage $\mathrm{HCC}^{3}$ For this reason, the EASL recommends a routine biopsy of these lesions regardless of 
LI-RADS classification as lower LI-RADS classes do not rule out the presence of HCC. It is therefore controversial as to how aggressively clinicians should diagnose HCC at a very early stage especially in LT candidates in the United States. It is less debatable in patients who would not be a candidate for $\mathrm{LT}$ because detection of very early stage HCC seems to improve clinical outcome.

\section{CONCLUSIONS}

In conclusion, detection of very early stage HCC is important as it leads to an excellent overall survival after curative treatment. However, diagnosis of HCC at a very early stage is technically challenging due to limited performance and risk of available diagnostic tests. The current organ allocation system in the United States made a huge impact on diagnostic approaches in patients with small indeterminate lesions seen in the surveillance ultrasound and subsequent diagnostic image tests. Use of liver biopsy for the detection of very early stage HCC is particularly controversial between the United States and European guideline. Novel blood-based biomarkers showed promising performances for the detection of very early stage HCC. Circulating DNA-based biomarkers in combination with images may improve the diagnostic performance and safety of surveillance tests, which can facilitate the diagnosis of very early stage $\mathrm{HCC}$ in the near future.

\section{Conflicts of Interest}

The author has no conflicts to disclose.

\section{REFERENCES}

1. Yang JD, Harmsen WS, Slettedahl SW, Chaiteerakij R, Enders FT, Therneau TM, et al. Factors that affect risk for hepatocellular carcinoma and effects of surveillance. Clin Gastroenterol Hepatol 2011;9:617-623.e1.

2. Yang JD, Kim WR, Park KW, Chaiteerakij R, Kim B, Sanderson SO, et al. Model to estimate survival in ambulatory patients with hepatocellular carcinoma. Hepatology 2012;56:614-621.

3. European Association for the Study of the Liver. EASL Clinical Practice Guidelines: management of hepatocellular carcinoma. J Hepatol 2018:69:182-236

4. Marrero JA, Kulik LM, Sirlin CB, Zhu AX, Finn RS, Abecassis MM, et al. Diagnosis, staging, and management of hepatocellular carcinoma: 2018 Practice Guidance by the American Association for the
Study of Liver Diseases. Hepatology 2018;68:723-750.

5. Omata M, Cheng AL, Kokudo N, Kudo M, Lee JM, Jia J, et al. AsiaPacific clinical practice guidelines on the management of hepatocellular carcinoma: a 2017 update. Hepatol Int 2017;11:317-370.

6. Silva MA, Hegab B, Hyde C, Guo B, Buckels JA, Mirza DF. Needle track seeding following biopsy of liver lesions in the diagnosis of hepatocellular cancer: a systematic review and meta-analysis. Gut 2008;57:1592-1596.

7. Rockey DC, Caldwell SH, Goodman ZD, Nelson RC, Smith AD; American Association for the Study of Liver Diseases. Liver biopsy. Hepatology 2009;49:1017-1044.

8. Forner A, Vilana R, Ayuso C, Bianchi L, Solé M, Ayuso JR, et al. Diagnosis of hepatic nodules $20 \mathrm{~mm}$ or smaller in cirrhosis: prospective validation of the noninvasive diagnostic criteria for hepatocellular carcinoma. Hepatology 2008;47:97-104.

9. Rich NE, Parikh ND, Singal AG. Hepatocellular carcinoma and liver transplantation: changing patterns and practices. Curr Treat Options Gastroenterol 2017;15:296-304.

10. Llovet JM, Brú C, Bruix J. Prognosis of hepatocellular carcinoma: the BCLC staging classification. Semin Liver Dis 1999;19:329-338.

11. Farinati F, Vitale A, Spolverato G, Pawlik TM, Huo TL, Lee YH, et al. Development and validation of a new prognostic system for patients with hepatocellular carcinoma. PLoS Med 2016;13:e1002006.

12. Llovet JM, Burroughs A, Bruix J. Hepatocellular carcinoma. Lancet 2003:362:1907-1917.

13. Roayaie S, Obeidat K, Sposito C, Mariani L, Bhoori S, Pellegrinelli $A$, et al. Resection of hepatocellular cancer $\leq 2 \mathrm{~cm}$ : results from two Western centers. Hepatology 2013;57:1426-1435.

14. Hasegawa K, Kokudo N, Makuuchi M, Izumi N, Ichida T, Kudo M, et al. Comparison of resection and ablation for hepatocellular carcinoma: a cohort study based on a Japanese nationwide survey. J Hepatol 2013;58:724-729.

15. Borzio M, Dionigi E, Rossini A, Marignani M, Sacco R, De Sio I, et al. External validation of the ITA.LI.CA prognostic system for patients with hepatocellular carcinoma: a multicenter cohort study. Hepatology 2018;67:2215-2225.

16. Sapisochin G, Facciuto M, Rubbia-Brandt L, Marti J, Mehta N, Yao FY, et al. Liver transplantation for "very early" intrahepatic cholangiocarcinoma: international retrospective study supporting a prospective assessment. Hepatology 2016;64:1178-1188.

17. Sapisochin G, Rodríguez de Lope C, Gastaca M, Ortiz de Urbina J, Suarez MA, Santoyo J, et al. "Very early" intrahepatic cholangiocarcinoma in cirrhotic patients: should liver transplantation be reconsidered in these patients? Am J Transplant 2014;14:660-667.

18. Sapisochin G, de Lope CR, Gastaca M, de Urbina JO, López-Andujar $R$, Palacios $F$, et al. Intrahepatic cholangiocarcinoma or mixed hepatocellular-cholangiocarcinoma in patients undergoing liver transplantation: a Spanish matched cohort multicenter study. Ann 
Surg 2014;259:944-952.

19. Sotiropoulos GC, Malagó M, Molmenti EP, Nadalin S, Radtke A, Brokalaki El, et al. Liver transplantation and incidentally found hepatocellular carcinoma in liver explants: need for a new definition? Transplantation 2006;81:531-535.

20. Senkerikova R, Frankova S, Sperl J, Oliverius M, Kieslichova E, Filipova $\mathrm{H}$, et al. Incidental hepatocellular carcinoma: risk factors and long-term outcome after liver transplantation. Transplant Proc 2014;46:1426-1429.

21. Yang JD, Addissie BD, Mara KC, Harmsen WS, Dai J, Zhang N, et al. GALAD score for hepatocellular carcinoma detection in comparison to liver ultrasound and proposal of GALADUS Score. Cancer Epidemiol Biomarkers Prev 2019;28:531-538.

22. Simmons O, Fetzer DT, Yokoo T, Marrero JA, Yopp A, Kono Y, et al. Predictors of adequate ultrasound quality for hepatocellular carcinoma surveillance in patients with cirrhosis. Aliment Pharmacol Ther 2017;45:169-177.

23. Tzartzeva K, Obi J, Rich NE, Parikh ND, Marrero JA, Yopp A, et al. Surveillance imaging and alpha fetoprotein for early detection of hepatocellular carcinoma in patients with cirrhosis: a meta-analysis. Gastroenterology 2018;154:1706-1718.e1.

24. Bruix J, Sherman M, Llovet JM, Beaugrand M, Lencioni R, Burroughs AK, et al. Clinical management of hepatocellular carcinoma. Conclusions of the Barcelona-2000 EASL conference. European Association for the Study of the Liver. J Hepatol 2001;35:421-430.

25. Bruix J, Sherman M; Practice Guidelines Committee, American Association for the Study of Liver Diseases. Management of hepatocellular carcinoma. Hepatology 2005;42:1208-1236.

26. Bruix J, Sherman M; American Association for the Study of Liver Diseases. Management of hepatocellular carcinoma: an update. Hepatology 2011;53:1020-1022.

27. Marrero JA, Feng Z, Wang Y, Nguyen MH, Befeler AS, Roberts LR, et al. Alpha-fetoprotein, des-gamma carboxyprothrombin, and lectinbound alpha-fetoprotein in early hepatocellular carcinoma. Gastroenterology 2009;137:110-118.

28. Tang A, Singal AG, Mitchell DG, Hecht EM, Fowler KJ, Kulik L, et al. Introduction to the Liver Imaging Reporting and Data System (LIRADS) for hepatocellular carcinoma. Clin Gastroenterol Hepatol 2019;17:1228-1238.

29. Khalili K, Kim TK, Jang HJ, Haider MA, Khan L, Guindi M, et al. Optimization of imaging diagnosis of $1-2 \mathrm{~cm}$ hepatocellular carcinoma: an analysis of diagnostic performance and resource utilization. J Hepatol 2011;54:723-728.

30. Pocha C, Dieperink E, McMaken KA, Knott A, Thuras P, Ho SB. Surveillance for hepatocellular cancer with ultrasonography vs. computed tomography -- a randomised study. Aliment Pharmacol Ther 2013;38:303-312.

31. Van Thiel DH, Yong S, Li SD, Kennedy M, Brems J. The development of de novo hepatocellular carcinoma in patients on a liver transplant list: frequency, size, and assessment of current screening methods. Liver Transpl 2004;10:631-637.

32. Kim SY, An J, Lim YS, Han S, Lee JY, Byun JH, et al. MRI with liverspecific contrast for surveillance of patients with cirrhosis at high risk of hepatocellular carcinoma. JAMA Oncol 2017;3:456-463.

33. Shah TU, Semelka RC, Pamuklar E, Firat Z, Gerber RD, Shrestha R, et al. The risk of hepatocellular carcinoma in cirrhotic patients with small liver nodules on MRI. Am J Gastroenterol 2006;101:533-540.

34. Roberts LR, Sirlin CB, Zaiem F, Almasri J, Prokop LJ, Heimbach JK, et al. Imaging for the diagnosis of hepatocellular carcinoma: a systematic review and meta-analysis. Hepatology 2018;67:401-421.

35. Atiq O, Tiro J, Yopp AC, Muffler A, Marrero JA, Parikh ND, et al. An assessment of benefits and harms of hepatocellular carcinoma surveillance in patients with cirrhosis. Hepatology 2017;65:1196-1205.

36. Kim HL, An J, Park JA, Park SH, Lim YS, Lee EK. Magnetic resonance imaging is cost-effective for hepatocellular carcinoma surveillance in high risk patients with cirrhosis. Hepatology 2019;69:1599-1613.

37. Forner A, Reig M, Bruix J. Alpha-fetoprotein for hepatocellular carcinoma diagnosis: the demise of a brilliant star. Gastroenterology 2009;137:26-29.

38. Chang TS, Wu YC, Tung SY, Wei KL, Hsieh YY, Huang HC, et al. Alpha-fetoprotein measurement benefits hepatocellular carcinoma surveillance in patients with cirrhosis. Am J Gastroenterol 2015;110:836-844; quiz 845.

39. Cho HJ, Kim B, Lee JD, Kang DR, Kim JK, Lee JH, et al. Development of risk prediction model for hepatocellular carcinoma progression of indeterminate nodules in hepatitis B virus-related cirrhotic liver. Am J Gastroenterol 2017;112:460-470.

40. Yang JD, Dai J, Singal AG, Gopal P, Addissie BD, Nguyen MH, et al. Improved performance of serum alpha-fetoprotein for hepatocellular carcinoma diagnosis in HCV cirrhosis with normal alanine transaminase. Cancer Epidemiol Biomarkers Prev 2017;26:1085-1092.

41. Sterling RK, Wright EC, Morgan TR, Seeff LB, Hoefs JC, Di Bisceglie $\mathrm{AM}$, et al. Frequency of elevated hepatocellular carcinoma (HCC) biomarkers in patients with advanced hepatitis C. Am J Gastroenterol 2012;107:64-74.

42. Choi J, Kim GA, Han S, Lee W, Chun S, Lim YS. Longitudinal assessment of three serum biomarkers to detect very early stage hepatocellular carcinoma. Hepatology 2019;69:1983-1994.

43. Tayob N, Lok AS, Do KA, Feng Z. Improved detection of hepatocellular carcinoma by using a longitudinal alpha-fetoprotein screening algorithm. Clin Gastroenterol Hepatol 2016;14:469-475.e2.

44. Lok AS, Sterling RK, Everhart JE, Wright EC, Hoefs JC, Di Bisceglie $A M$, et al. Des-gamma-carboxy prothrombin and alpha-fetoprotein as biomarkers for the early detection of hepatocellular carcinoma. Gastroenterology 2010;138:493-502.

45. Berhane S, Toyoda H, Tada T, Kumada T, Kagebayashi C, Satomura 
S, et al. Role of the GALAD and BALAD-2 serologic models in diagnosis of hepatocellular carcinoma and prediction of survival in patients. Clin Gastroenterol Hepatol 2016;14:875-886.e6.

46. Kisiel JB, Dukek BA, Kanipakam RVSR, Ghoz HM, Yab TC, Berger CK, et al. Hepatocellular carcinoma detection by plasma methylated DNA: discovery, phase I pilot, and phase II clinical validation. Hepatology 2019;69:1180-1192.

47. Yang JD, Roberts LR. Hepatocellular carcinoma: a global view. Nat Rev Gastroenterol Hepatol 2010;7:448-458.

48. Mehta N, Heimbach J, Harnois DM, Sapisochin G, Dodge JL, Lee D, et al. Validation of a risk estimation of tumor recurrence after transplant (RETREAT) score for hepatocellular carcinoma recurrence after liver transplant. JAMA Oncol 2017;3:493-500.

49. Yang JD, Larson JJ, Watt KD, Allen AM, Wiesner RH, Gores GJ, et al. Hepatocellular carcinoma is the most common indication for liver transplantation and placement on the waitlist in the United States. Clin Gastroenterol Hepatol 2017;15:767-775.e3.

50. Wiesner R, Edwards E, Freeman R, Harper A, Kim R, Kamath $P$, et al. Model for end-stage liver disease (MELD) and allocation of donor livers. Gastroenterology 2003;124:91-96.

51. Kamath PS, Wiesner RH, Malinchoc M, Kremers W, Therneau TM, Kosberg $\mathrm{CL}$, et al. A model to predict survival in patients with endstage liver disease. Hepatology 2001;33:464-470.

52. Heimbach JK, Hirose R, Stock PG, Schladt DP, Xiong H, Liu J, et al. Delayed hepatocellular carcinoma model for end-stage liver disease exception score improves disparity in access to liver transplant in the
United States. Hepatology 2015;61:1643-1650.

53. Mehta N, Sarkar M, Dodge JL, Fidelman N, Roberts JP, Yao FY. Intention to treat outcome of $\mathrm{T} 1$ hepatocellular carcinoma with the "wait and not ablate" approach until meeting T2 criteria for liver transplant listing. Liver Transpl 2016;22:178-187.

54. Tang A, Bashir MR, Corwin MT, Cruite I, Dietrich CF, Do RKG, et al. Evidence supporting LI-RADS major features for CT- and MR imaging-based diagnosis of hepatocellular carcinoma: a systematic review. Radiology 2018;286:29-48.

55. Chernyak V, Fowler KJ, Kamaya A, Kielar AZ, Elsayes KM, Bashir $M R$, et al. Liver Imaging Reporting and Data System (LI-RADS) version 2018: imaging of hepatocellular carcinoma in at-risk patients. Radiology 2018;289:816-830.

56. van der Pol CB, Lim CS, Sirlin CB, McGrath TA, Salameh JP, Bashir $M R$, et al. Accuracy of the Liver Imaging Reporting and Data System in computed tomography and magnetic resonance image analysis of hepatocellular carcinoma or overall malignancy-a systematic review. Gastroenterology 2019;156:976-986.

57. Terzi E, lavarone M, Pompili M, Veronese L, Cabibbo G, Fraquelli M, et al. Contrast ultrasound LI-RADS LR-5 identifies hepatocellular carcinoma in cirrhosis in a multicenter restropective study of 1,006 nodules. J Hepatol 2018;68:485-492.

58. Aubé C, Oberti F, Lonjon J, Pageaux G, Seror O, N'Kontchou G, et al. EASL and AASLD recommendations for the diagnosis of HCC to the test of daily practice. Liver Int 2017;37:1515-1525. 